\title{
The angular response of ultrathin film organic solar cells
}

\author{
D. Cheyns, ${ }^{\text {a),b) }}$ B. P. Rand, B. Verreet, ${ }^{\text {b) }}$ J. Genoe, J. Poortmans, ${ }^{\text {b) }}$ and \\ P. Heremans ${ }^{\text {b) }}$ \\ IMEC vzw, Kapeldreef 75, 3001 Leuven, Belgium
}

(Received 17 March 2008; accepted 2 June 2008; published online 20 June 2008)

\begin{abstract}
We study the effect of oblique incident light on the optical interference effects and photocurrent of organic planar heterojunction solar cells. We find that the thin layers used in organic solar cells induce a complex light distribution within the device, further altered by incident angle. The responsivity can increase as a function of angle to values up to $15 \%$ larger than at normal incidence, and optical simulations are shown to match these trends. Furthermore, we show that the outdoor performance at different tilting angles is higher than expected, which improves the attractiveness of organic solar cells for building-integrated applications. (C) 2008 American Institute of Physics.

[DOI: 10.1063/1.2949745]
\end{abstract}

The total active layer thickness of today's best performing organic solar cells (OSCs) is on the order of 50-200 nm. This is due to the intrinsic optical and electrical properties of organic solids, in particular high absorption coefficients $\left(\sim 10^{5} \mathrm{~cm}^{-1}\right)$ as well as low charge carrier mobilities $\left(10^{-6}-10^{-2} \mathrm{~cm}^{2} / \mathrm{V} \mathrm{s}\right)$. One consequence of the use of these ultrathin organic films is the presence of a nonuniform light intensity distribution that depends on layer thickness and the complex index of refraction of the materials. Careful design is necessary to account for these optical interference effects. A method to calculate the standing waves in thin layers, and which has been widely used to optimize OSCs under normal light incidence, ${ }^{1-5}$ is the transfer matrix method (TMM). These optical simulations assist in positioning the charge generating donor-acceptor heterojunction at the point of highest light intensity. Here, we investigate the angular response of OSCs, because this also needs to be considered to optimize devices for realistic applications, where the lowcost requirements would almost certainly exclude the possibility of solar tracking systems.

Previous measurements carried out on bare and encapsulated thick inorganic solar cells showed a clear decrease in the responsivity (i.e., short-circuit current divided by incident light power) when diverging from normal light incidence. ${ }^{6,7}$ In contrast to those solar cells without optical interference (devices with absorber thickness much greater than the wavelength of incident light), the responsivity of ultrathin OSCs can increase with more oblique incident angles, ${ }^{3,8}$ peaking even at angles up to $60^{\circ}$. As a result, taking into account Lambert's cosine law, the generated current is less affected by the incident light angle. This paper uses experiments and simulations on planar heterojunction solar cells incorporating various materials with different absorption spectra to illustrate the angle dependent carrier generation as well as the influence of the absorption range. Subsequently, we extend our study to the outdoor use of OSCs, showing that the cumulative year current for a properly designed cell can be higher than expected.

Indium tin oxide coated glass substrates (Merck, $\rho$ $<20 \Omega / \square$ ) are cleaned using soap, de-ionized water, ac-

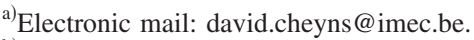

${ }^{b)}$ Also at ESAT, Katholieke Universiteit Leuven, Kasteelpark Arenberg 10, B-3001 Leuven, Belgium.
}

etone, isopropanol, and $\mathrm{UV} / \mathrm{O}_{3}$ treatment. The organic materials boron-sub-phthalocyanine (SubPc), pentacene, fullerene $\left(\mathrm{C}_{60}\right)$, and bathocuproine $(\mathrm{BCP})$ are purchased from Aldrich, and purified once using vacuum thermal gradient sublimation. All layers are deposited in ultrahigh vacuum (base pressure $<1 \times 10^{-8}$ Torr) without breaking vacuum, except for mounting shadow masks to evaporate a $3 \mathrm{~mm}^{2}$ cathode ( $\mathrm{Al}$ or $\mathrm{Yb}$ ) in an $\mathrm{N}_{2}$ atmosphere.

The optical properties of the materials used in this study (organics, metals, glass, etc.) are determined using ellipsometry (SOPRA, GES5) and verified by comparing to UV-Vis absorption data, and these are shown for the absorbing organic layers in Fig. 1. Anisotropic complex indices of refraction are known for some organic materials. Large anisotropy or thick layers can induce a Poynting vector that is not parallel to the wave vector, reducing the correctness of TMM. However, the growth of the used organic molecules do not show large crystallinity, and the layer thicknesses are small. Anisotropy will induce only minor changes to the light distribution in the layer stack, and for this reason, we can neglect the optical anisotropy.

The optical simulations are conducted using TMM, and the equations found in literature are adapted for obliquely incident light. ${ }^{1,9}$ In this case, unpolarized light is split into $s$

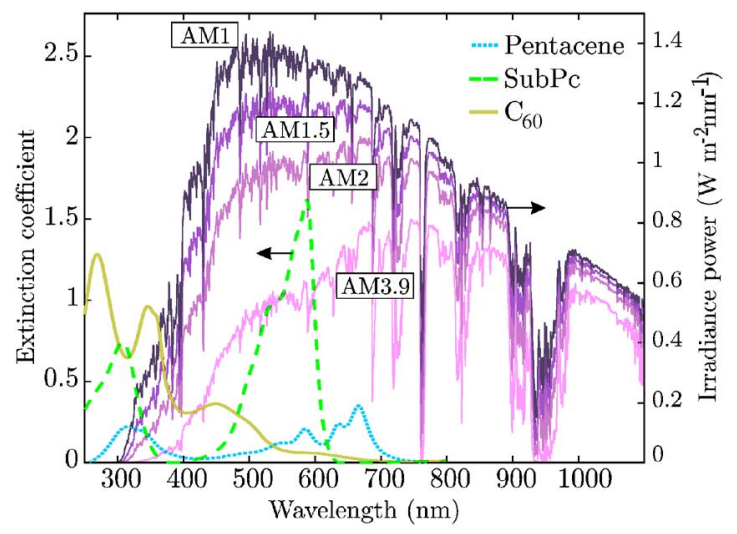

FIG. 1. (Color online) Extinction coefficients of pentacene (dotted line), SubPc (dashed line), and $\mathrm{C}_{60}$ (straight line). On the right axis, solar spectra for different zenith angles are plotted and only direct irradiation is included. The various AM direct spectra and corresponding zenith angles are AM1 $=0^{\circ}, \mathrm{AM} 1.5=48.2^{\circ}, \mathrm{AM} 2=60^{\circ}$, and $\mathrm{AM} 3.9=75^{\circ}$. 


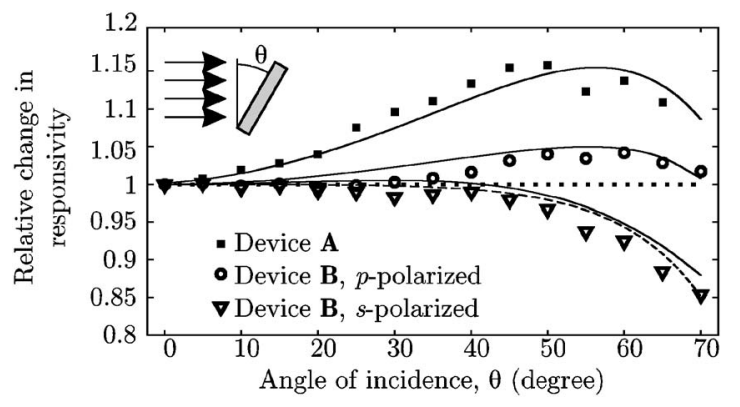

FIG. 2. Experimental data (marks) and corresponding simulations (lines) of devices $\mathbf{A}$ and $\mathbf{B}$. The change in responsivity, normalized to the responsivity at normal incidence, is plotted as a function of the incident light angle. For device $\mathbf{B}$, the effect of both $p$ - $(\mathbf{O})$ and $s$-polarized $(\nabla)$ light is measured and simulated. The dotted line corresponds to Lambert's cosine law, the expected decrease in incident light intensity per illuminated area vs angle. The dashed line corresponds to the case where only the air-glass reflection is taken into account as affecting the responsivity.

and $p$ polarizations (perpendicular to and in the plane of incidence, respectively), as they behave differently. From the generated exciton density in the layer structure, we calculate the steady-state exciton density and the number of excitons diffusing to the donor-acceptor interface. We assume unity exciton dissociation and charge collection efficiencies, or that the number of excitons reaching the interface is equal to the short-circuit current $\left(I_{\mathrm{SC}}\right)$ generated by the device. By doing this, we obtain an external quantum efficiency (EQE) for every incident wavelength $\lambda$ and angle $\theta$. The generated current is then calculated by convoluting EQE and the solar spectrum.

To compare the effect of angular dependency on devices with different absorption profiles, we investigate two device structures. Device A consists of $50 \mathrm{~nm}$ pentacene/30 nm $\mathrm{C}_{60} / 100 \mathrm{~nm} \mathrm{Yb}$, while the structure of device $\mathbf{B}$ is $20 \mathrm{~nm}$ $\mathrm{SubPc} / 35 \mathrm{~nm} \mathrm{C} 60 / 10 \mathrm{~nm} \mathrm{BCP/100} \mathrm{nm} \mathrm{Al.} \mathrm{The} \mathrm{devices} \mathrm{are}$ first characterized with a $1000 \mathrm{~W}$ solar simulator (L.O.T.Oriel) equipped with filters to obtain $100 \mathrm{~mW} / \mathrm{cm}^{2}$ AM1.5D spectrum. Device $\mathbf{A}$ has an open-circuit voltage $\left(V_{\mathrm{OC}}\right)$ of $340 \mathrm{mV}, I_{\mathrm{SC}}=3.0 \mathrm{~mA} / \mathrm{cm}^{2}$, and a fill factor $(\mathrm{FF})$ of $55 \%$, reaching a power conversion efficiency $(\eta)$ of $0.56 \%$, while the characteristics of device $\mathbf{B}$ are $V_{\mathrm{OC}}=640 \mathrm{mV}$, $I_{\mathrm{SC}}=4.15 \mathrm{~mA} / \mathrm{cm}^{2}, \mathrm{FF}=60 \%$, with $\eta=1.6 \%$.

The angular dependent measurements are carried out in an inert $\mathrm{N}_{2}$ atmosphere using simulated solar light from a $100 \mathrm{~W}$ Xe arc lamp (L.O.T.-Oriel), with optical filters to correct for the AM1.5D spectrum. The same light spot was used to illuminate the solar cells under different $\theta$, avoiding fluctuations in current due to homogeneous light distribution of the light source. The relative error on the angle is less than $0.5^{\circ}$. During the measurement we rotate the device with respect to a fixed light source and, as a consequence, the incident light power per unit area decreases according to Lambert's cosine law. As we are interested in the change in responsivity, we correct for this decrease in incident light. The relative change in responsivity is plotted in Fig. 2, where the dotted line represents Lambert's cosine law, and the dashed line the effect of the reflection at the air-glass interface.

The responsivity of device $\mathbf{A}$ increases along with $\theta$, and reaches a peak at $50^{\circ}$, where there is a $15 \%$ increase over that at normal incidence and, in fact, we observe this increase to persist for $\theta$ up to $70^{\circ}$. Although the trend for $p$-polarized Downloaded 23 Jun 2008 to 146.103.254.11. Redistribution subject

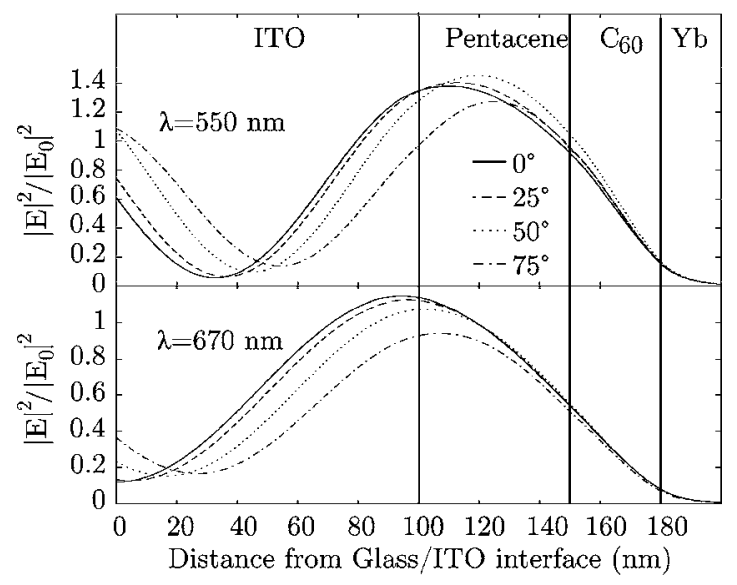

FIG. 3. Normalized modulus squared of the optical electrical field $\left(|E|^{2} /\left|E_{0}\right|^{2}\right)$ inside device $\mathbf{A}$ for two wavelengths, $550 \mathrm{~nm}$ (upper panel) and $670 \mathrm{~nm}$ (lower panel), and for incident light angles $0^{\circ}, 25^{\circ}, 50^{\circ}$, and $75^{\circ}$.

light on device $\mathbf{B}$ is the same, the decrease for $s$-polarized light compensates this increase. The major contribution to the difference for the two polarizations is due to the reflection of incident light at the air-glass interface. Brewster's angle for this interface, that is, the angle at which $p$-polarized light has a $100 \%$ transmission, is $56^{\circ}$, which coincides with the maximum of the responsivity. The results of the TMM shown as solid curves are in very close agreement with the experimental data, validating the assumption that possible optical anisotropy has only a minor influence on the light distribution.

The increase in responsivity at different $\theta$ can be understood by considering the standing light wave inside the device structure. We plot the electric field intensity within device A for two different wavelengths $(\lambda=670$ and $550 \mathrm{~nm}$, corresponding to two absorption peaks of pentacene) and various $\theta$ in Fig. 3. Two effects are particularly noteworthy: the absolute maximum of the electric field changes and the position of this maximum shifts. The first effect is difficult to predict analytically. The reflection at the air-glass interface increases, so a decrease in the maximum is expected, as observed at $\lambda=670 \mathrm{~nm}$. This is not the case for $\lambda=550 \mathrm{~nm}$, suggesting that the changing reflections at other surfaces can overcome this decrease. The second effect, that of the position of the maximum inside the device, follows a specific trend for planar interfaces: when $\theta$ is increased, the maximum of the standing wave will shift closer to the top contact, following a cosine behavior. This position is determined by the path length from the top contact, which acts as a mirror and will thus produce the standing wave. In the case of device $\mathbf{A}$, the shift towards the contact positions the maximum closer to the donor-acceptor interface. As the exciton dissociation occurs here, this produces the increase in current shown in Fig. 2. The effect depends strongly on the chosen thickness of the layers and on material choice, as the layer structure of device $\mathbf{B}$ shows less overall change in responsivity.

Now, with the knowledge of how the responsivity changes as a function of the incident angle, we can calculate the outdoor performance of an OSC in a fixed position. For this calculation, we need to consider the position of the sun, which will determine the solar spectrum as well as the incident angle of the light on the device. To describe the position of the sun relative to an object on Earth's surface, the zenith o AIP license or copyright; see http://apl.aip.org/apl/copyright.jsp 


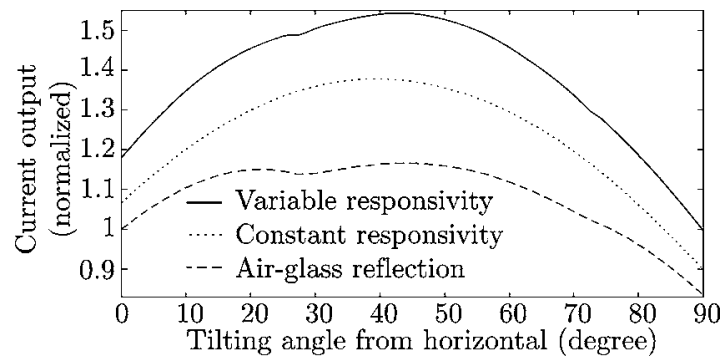

FIG. 4. Normalized simulated current output vs the tilting angle of a solar cell for a latitude of $50^{\circ}$, with the normal to the solar cell pointing to the south. Three cases are plotted: the variable responsivity of device $\mathbf{A}$, a constant responsivity, and a decreasing responsivity due to the air-glass interface.

angle $(Z)$ and the hour angle $(\omega)$ are used. ${ }^{10}$ The zenith angle depends on the latitude $(\Lambda)$ and declination $(\delta)$ of the position on Earth under investigation,

$$
\cos (Z)=\sin (\Lambda) \sin (\delta)+\cos (\Lambda) \cos (\delta) \cos (\omega),
$$

where $\omega$ measures the relative position of the sun as it moves in the plane of the apparent orbit of the sun.

The air mass (AM), or the effective travel distance of the light through Earth's atmosphere, subsequently depends on $Z$. A higher AM index is reflected by more absorption and diffraction of the light in the atmosphere, taken into account by the atmospheric extinction (AE) coefficient. From the standard spectrum for AM0 and AM1.5D, ${ }^{11}$ we calculate $\mathrm{AE}$ for each wavelength and use this value to estimate the spectra for other AM indices. Some of the resulting spectra can be seen in Fig. 1. Other changes in the solar spectrum (e.g., clouds) and the diffuse circumsolar component are disregarded. A clear disadvantage of the organic materials chosen here is that the absorption spectra overlap with the solar spectrum in regions with a high AE. Materials with a more redshifted absorption spectrum would be less affected.

For simplicity, we only calculate the performance of solar cells with the normal of the surface pointing to the south and a changing tilt angle $T$. The incident angle $\theta$ on the solar cell can be expressed as a function of the other angles, viz.

$$
\begin{aligned}
\cos (\theta)= & \cos (Z) \cos (T)-\sin (\delta) \cos (\Lambda) \sin (T) \\
& +\cos (\delta) \sin (\Lambda) \sin (T) \cos (\omega) .
\end{aligned}
$$

By combining the calculation of the EQE for each incident angle with the exact cell-sun angle and AM spectra throughout the day and year, in Fig. 4 we obtain a cumulative year current for device $\mathbf{A}$, which will be directly related to the maximum power produced in a year. In this case, the optimal tilting angle is at $41^{\circ}$. In order to have an idea of the effect of the angular response, we compare two other cumulative year currents. The first case is the case of a constant spectral response as a function of the angle or Lambert's cosine law. The second case considers the reflection at the air-glass interface, which is the case for thick solar cells where optical interference effects are not present. All the graphs are normalized to this last case, with the device in horizontal position. The initial gain for horizontally positioned solar cells is close to $20 \%$ for device A. While the maximum output is obtained at around $40-45^{\circ}$ for all cases, the gain is the highest for the real situation of device $\mathbf{A}$. Although the gain is the lowest for vertically oriented solar cells $\left(90^{\circ}\right)$, there is still a $15 \%$ increase compared to the case with the air-glass reflection.

In conclusion, we show that the responsivity of organic planar heterojunction solar cells depends strongly on the incident angle. In the case of a pentacene- $\mathrm{C}_{60}$ solar cell, the responsivity increases up to $15 \%$, peaking at incident angles of $50^{\circ}-55^{\circ}$ and making the need for complicated antireflection coatings low. The implication on the design of tandem cells is significant, as a matching current in multiple series connected subcells is needed, and this current should be maintained for incident angle and solar spectrum. Also, we found that the angle dependent responsivity is highly relevant when the outdoor performance of these cells are evaluated. In our case, an underestimation of up to $40 \%$ would occur if this effect is not taken into account. The gain by this effect is visible for all tilting angles of a fixed solar cell, making these cells candidates for building-integrated applications.

The authors thank K. Weerts and C. Girotto for help with the optical setup and J. Cuypers for useful discussions. Part of this work is supported by the European-funded Project FACESS (ICT No. 215271).

${ }^{1}$ L. A. A. Pettersson, L. S. Roman, and O. Inganäs, J. Appl. Phys. 86, 487 (1999).

${ }^{2}$ H. Hoppe, N. Arnold, N. S. Sariciftci, and D. Meissner, Secur. Manage. 80, 105 (2003).

${ }^{3}$ G. Dennler, K. Forberich, M. C. Scharber, C. J. Brabec, I. Tomiš, K. Hingerl, and T. Fromherz, J. Appl. Phys. 102, 054516 (2007).

${ }^{4}$ S. Yoo, B. Domercq, and B. Kippelen, Appl. Phys. Lett. 85, 5427 (2004). ${ }^{5}$ P. Peumans, A. Yakimov, and S. R. Forrest, J. Appl. Phys. 93, 3693 (2003).

${ }^{6}$ J. L. Balenzategui and F. Chenlo, Sol. Energy Mater. Sol. Cells 86, 53 (2005).

${ }^{7}$ H. Sai, H. Fujii, K. Arafune, Y. Ohshita, Y. Kanamori, H. Yugami, and M. Yamaguchi, Jpn. J. Appl. Phys., Part 1 46, 3333 (2007).

${ }^{8}$ M. Niggemann, M. Glatthaar, P. Lewer, C. Müller, J. Wagner and A. Gombert, Thin Solid Films 511, 628 (2006).

${ }^{9}$ R. M. A. Azzam and N. M. Bashara, Ellipsometry and Polarized Light (Elsevier, Amsterdam, 1987).

${ }^{10}$ J. A. Duffie and W. A. Beckman, Solar Engineering of Thermal Processes (Wiley, New York, 1980).

${ }^{11}$ NREL Reference Solar Spectral Irradiance, http://rredc.nrel.gov/solar/ spectra.am $1.5 /$ 